\title{
Resistência biológica da madeira de eucalipto tratada com CCA-C a cupins subterrâneos em laboratório
}

\author{
Biological resistance to subterranean termites of \\ eucalypt wood treated with CCA-C in laboratory tests
}

\author{
Dercilio Junior Verly Lopes ${ }^{1}$, Juarez Benigno Paes ${ }^{2}$, Ivaldo Pontes Jankowsky ${ }^{3}$, \\ Graziela Baptista Vidaurre ${ }^{4}$ e Pedro Gutemberg Alcântara Segundinho ${ }^{4}$
}

\begin{abstract}
Resumo
O objetivo do trabalho foi avaliar a resistência biológica de moirões de Corymbia torelliana e Eucalyptus grandis $\times$ Eucalyptus urophylla, tratados com CCA-C, ao cupim Nasutitermes corniger em testes de laboratório. Para o tratamento dos moirões empregou-se o processo de célula cheia (Bethell). Os moirões tinham 2,20 metros de comprimento e classes diamétricas de 8 a 10; 10 a 12 e 12 a $14 \mathrm{~cm}$. Foram sorteados oito moirões por classe e espécie florestal, e seis foram tratados e dois não tratados (controle). Foi cortado um disco de 2,0 cm de espessura, a $0,50 \mathrm{~m}$ da base de cada moirão (não tratado), para avaliar a massa específica básica e as porcentagens de cerne e alburno e, a penetração de cobre (moirão tratado). De cada disco (moirão tratado) foram retiradas quatro amostras da periferia até $1,5 \mathrm{~cm}$ (posição 1) e de 1,5 a $3,0 \mathrm{~cm}$ (posição 2) para avaliar a retenção e o grau de deterioração provocado nos moirões pelo cupim $N$. corniger. Foram realizados ensaios de alimentação forçada e de preferência alimentar. As maiores perdas de massa nos ensaios de alimentação forçada e de preferência alimentar foram para a madeira de $C$. torelliana. Para as madeiras tratadas os cupins sobreviveram 13 e 9 dias, para o C. torelliana e $E$. grandis $x$ E. urophylla, respectivamente. Os melhores resultados de resistência biológica foram encontrados para a madeira tratada de E. grandis $\times$ E. urophylla em ambos os ensaios.
\end{abstract}

Palavras-chave: Ensaios biológicos, moirões, Corymbia torelliana, Eucalyptus grandis x Eucalyptus urophylla.

\begin{abstract}
This work aimed to evaluate the biological resistance of Corymbia torelliana and Eucalyptus grandis $\mathrm{x}$ Eucalyptus urophylla wood treated with CCA-C, against the termite Nasutitermes corniger in laboratory tests. For the treatment of fence posts the full-cell process (Bethell) was employed. The fence posts measured 2.20 meters in length and diametrical classes were of 8 to $10 ; 10$ to 12 and 12 to $14 \mathrm{~cm}$. Thus eight fence posts per class and forest species were selected, and six were treated and two untreated (control). A disk of $2.0 \mathrm{~cm}$ thickness at $.50 \mathrm{~m}$ from the base was cut from each fence post (untreated), to evaluate the basic density and the heartwood and sapwood percentages and the penetration of copper (treated fence posts). Of each disk (treated fence posts) four samples were obtained from the periphery to $1.5 \mathrm{~cm}$ (position 1) and 1.5 to $3.0 \mathrm{~cm}$ (position 2) to evaluate the retention and degree of deterioration caused by $N$. corniger termite in the fence post. Tests were carried out with no choice feeding and choice feeding tests. The greatest mass losses in the no choice feeding and choice feeding tests were those with $C$. torelliana wood. In treated wood termites survived 13 and 9 days to the $C$. torelliana and $E$. grandis $\times E$. urophylla woods, respectively. The best results of biological resistance to termites were found for the $E$. grandis $\times E$. urophylla treated wood in both tests.
\end{abstract}

Keywords: Biological tests, fence posts, Corymbia torelliana, Eucalyptus grandis x Eucalyptus urophylla.

\footnotetext{
${ }^{1}$ Doutorando em Recursos Florestais. MSU - Mississippi State University. 75 B.S. Hood Dr, MS 39762, Starkville, Mississipi, EUA. E-mail: dvl23@msstate.edu.

2Professor Titular do Departamento de Ciências Florestais e da Madeira. UFES - Universidade Federal do Espírito Santo / CCAE - Centro de Ciências Agrárias e Engenharias. Av. Gov. Lindemberg, 316 - Caixa-postal: 16 - 29550-000 - Jeronimo Monteiro, ES, Brasil. E-mail: jbp2@uol.com.br.

3Professor Associado do Departamento de Ciências Florestais. USP - Universidade de São Paulo / ESALQ - Escola Superior de Agricultura "Luiz de Queiroz". Av. Pádua Dias, 11 - Caixa Postal 09 - 13418-900 - Piracicaba, SP, Brasil. E-mail: jankowsky@usp.br.

${ }^{4}$ Professor(a) Adjunto(a) no Departamento de Ciências Florestais e da Madeira. UFES - Universidade Federal do Espírito Santo / CCAE - Centro de Ciências Agrárias e Engenharias. Av. Gov. Lindemberg, 316 - Caixa-postal: 16 - 29550-000 - Jeronimo Monteiro, ES, Brasil. E-mail: grazividaurre@gmail.com; p_gutemberg2001@yahoo.com.br.
}

Sci. For., Piracicaba, v. 45, n. 115, p. 467-479, set. 2017 DOI: dx.doi.org/10.18671/scifor.v45n115.05 


\section{INTRODUÇÃO}

A finalidade da proteção da madeira com produtos químicos, também chamada de tratamento da madeira consiste em fornecer proteção a longo prazo contra os efeitos dos organismos xilófagos como fungos, insetos e brocas marinhas, estendendo desta forma a vida útil dos produtos a base de madeira.

No Brasil a indústria de preservação de madeiras, no ano de 2014, utilizou 1,82 milhões de metros cúbicos de madeira, de um total de 190,03 milhões de metros cúbicos empregados por todos os segmentos que usam a madeira de reflorestamento como fonte de matéria-prima, de acordo com a Indústria Brasileira de Árvores - IBÁ (2015). O consumo de madeira tratada no ano de 2014, em relação ao ano de 2013, foi semelhante, mesmo assim, quando se consideram todos os segmentos, houve um aumento de 0,026\%, o que equivaleu a 4.756.534 metros cúbicos (IBÁ, 2014).

Ao analisar ambos os relatórios da IBÁ $(2014,2015)$ não se tem referência sobre qual produto preservativo e nem sobre qual método de preservação (célula cheia ou célula vazia) foi utilizado na impregnação da madeira. Entretanto, o arseniato de cobre cromatado, tipo C, CCA-C, é o mais difundido em todo o mundo (RICHARDSON, 1993), em virtude de sua composição química contra fungos e cupins. Desta forma pode-se inferir que, em ambos os anos, grande parte da madeira foi tratada com o CCA-C.

A madeira tratada de eucalipto, principalmente a destinada a moirões é proveniente de lenho jovem, com pouca durabilidade natural, inclusive o cerne, e também apresenta tensões de crescimento, que geram rachaduras nas peças, depois de secas, expondo partes internas, não bem protegidas pelo produto químico utilizado. Aliado a isto, há casos em que a mesma não atinge a penetração e retenções recomendadas (PAES et al., 2012), estando sujeita ao ataque de fungos e insetos xilófagos.

Os insetos xilófagos são representados, principalmente, pelas ordens Isóptera e Coleóptera. Dentre estas, a primeira, composta pelas térmitas ou cupins, é a que representa o maior risco à utilização da madeira. Os cupins são, dentre os insetos, os mais severos agentes destruidores da madeira, os de solos, ou subterrâneos, são responsáveis pelos maiores volumes de perda de madeira no mundo (CLAUSEN, 2010).

A importância do controle de térmitas, em geral, está embasada no prejuízo econômico que estes organismos causam. Estimativas dos Estados Unidos da América relatam que há gasto da ordem de US $\$ 2,5$ bilhões com controle de térmitas e, deste valor, 66 a $80 \%$ são destinados apenas para controle de térmitas subterrâneas, sendo estes ataques evidentes em residências (BAKER; MARCHOSKY JUNIOR, 2005). No Brasil não há estatísticas sobre o ataque de cupins em residências e prédios públicos. Apenas alguns diagnósticos, a exemplo dos realizados por Eleotério (2000) e Silva (2008), mas, acredita-se, em decorrências das condições edafoclimáticas do País, que o consumo de madeira, por estes insetos, seja expressivo.

Na área de preservação de madeiras tem-se carência em investigações científicas sobre a eficácia dos parâmetros do tratamento preservativo industrial com pressão na durabilidade de madeiras. Desta forma, levando em consideração a importância do tema, o objetivo deste trabalho foi avaliar a resistência biológica de moirões de Corymbia torelliana e Eucalyptus grandis x Eucalyptus urophylla, tratados com CCA-C, ao cupim Nasutitermes corniger em testes de laboratório.

\section{MATERIAL E MÉTODOS}

\section{Espécies utilizadas, amostragem, teor de cerne e tratamento dos moirões}

Foram utilizados moirões de Corymbia torelliana e Eucalyptus grandis $\mathrm{x}$ Eucalyptus urophylla provenientes de uma empresa de tratamento preservativo industrial com pressão do norte do estado do Espírito Santo. As espécies florestais utilizadas são as mais tratadas e comercializadas na região. Os dados referentes aos povoamentos florestais que originaram os moirões encontram-se na Tabela 1.

Os moirões avaliados tinham 2,20 metros de comprimento, compreendidos nas classes diamétricas de 8 a $10 \mathrm{~cm} ; 10$ a $12 \mathrm{~cm} \mathrm{e} 12$ a $14 \mathrm{~cm}$. Destes foram sorteados oito moirões para cada classe diamétrica, em que seis foram tratados com CCA-C, e dois mantidos não tratados (controle). Retirou-se, de cada um deles, um disco de 2,0 cm de espessura a 0,50 $\mathrm{m}$ da base (Figura 1), para avaliar a massa específica básica, conforme metodologia descrita por Vital (1984), as porcentagens de cerne e alburno (moirão não tratado), a penetração de cobre e a retenção de CCA-C (moirão tratado). 
Tabela 1. Dados referentes ao plantio da espécie e híbrido.

Table 1. Information on planting of species and hybrid.

\begin{tabular}{lccccc}
\hline Madeiras & Idade (anos) & Altitude $(\mathbf{m})$ & Latitude & Longitude & Espaçamento do Plantio (m) \\
\hline C. torelliana & 11 & 109 & $18^{\circ} 27^{\prime} 37^{\prime \prime} \mathrm{S}$ & $40^{\circ} 10^{\prime} 36^{\prime \prime} \mathrm{O}$ & $3,00 \times 2,00$ \\
E. grandis x E. urophylla & 9 & 101 & $18^{\circ} 28^{\prime} 21^{\prime \prime} \mathrm{S}$ & $40^{\circ} 09^{\prime} 00^{\prime \prime} \mathrm{O}$ & $3,00 \times 2,00$ \\
\hline
\end{tabular}

De cada disco (moirão tratado) foram retiradas quatro amostras da periferia até $1,5 \mathrm{~cm}$ (posição 1) e de 1,5 a 3,0 cm (posição 2), em lados opostos, passando pela medula, a fim de analisar a retenção e o grau de deterioração provocado pelos cupins nos moirões. Na Figura 1 é exemplificada a retirada do disco e a confecção dos corpos de prova.

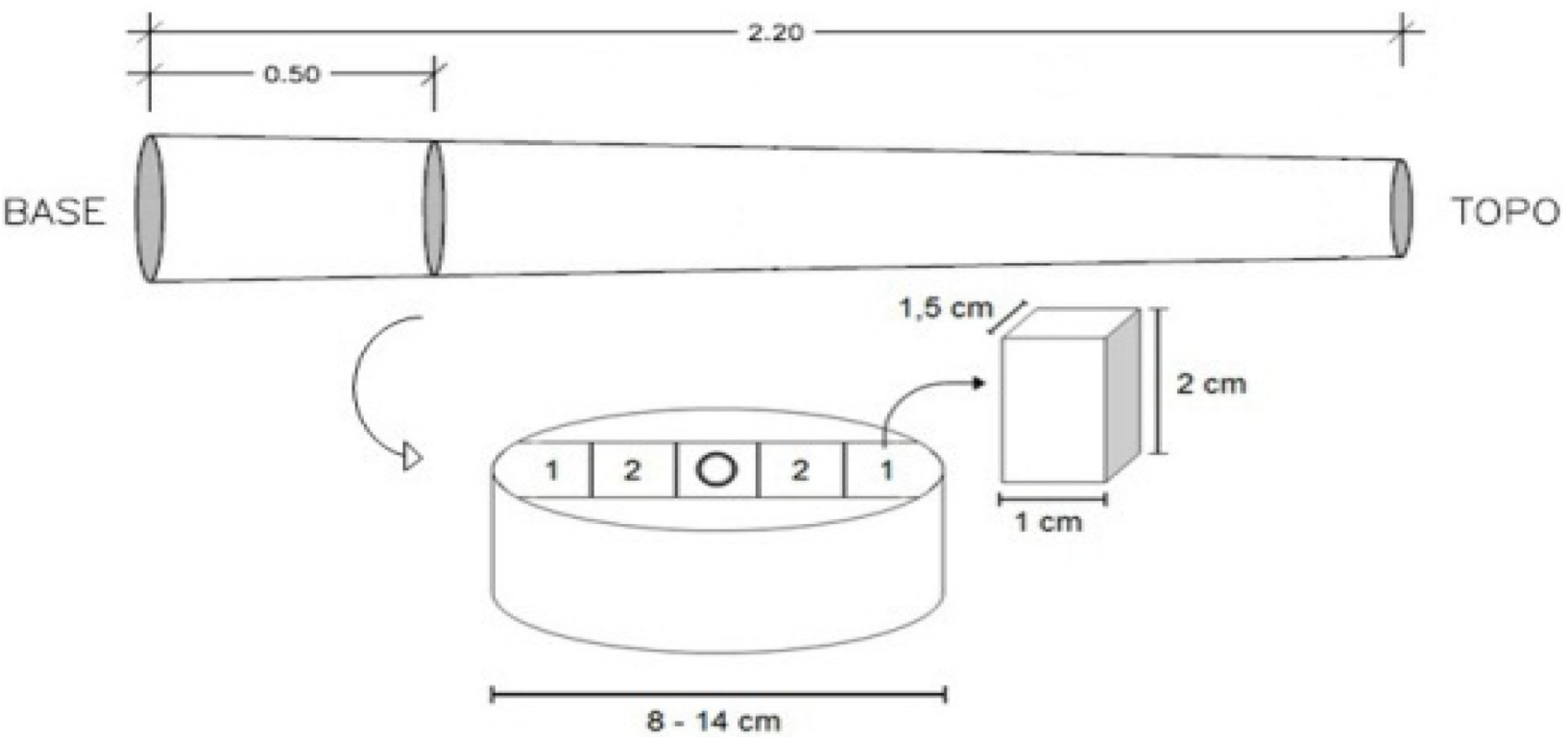

Figura 1. Posição onde foi retirado o disco do moirão e obtenção dos corpos de prova.

Figure 1. Position where fence post disk was removed and obtaining of test samples.

O teor de cerne e alburno foi determinado nos moirões não tratados por meio de discos obtidos na zona de afloramento $(0,50 \mathrm{~m})$. Nesta posição foram mensurados os perímetros externos e, também, demarcados e mensurados os limites do cerne e do alburno, em cada peça, para determinação da sua área, com uso de régua de $0,1 \mathrm{~cm}$ de precisão. Para tanto, os discos foram lixados a fim de melhor evidenciar o limite entre o cerne e o alburno.

O tratamento preservativo industrial com pressão foi conduzido seguindo as condições de vácuo inicial e final de $550 \mathrm{mmHg}$, durante 30 minutos, pressão nominal de tratamento de $11 \mathrm{kgf} \mathrm{cm}^{-2}$, durante uma hora, ao ser utilizada uma solução com $2 \%$ de ingredientes ativos (i.a) do produto arseniato de cobre cromatado, tipo C, CCA-C, calculada em base óxida.

\section{Penetração e retenção do produto na madeira}

A determinação da penetração do CCA teve como base o elemento cobre presente na madeira tratada. Portanto, foi realizada a análise colorimétrica, seguindo as recomendações da Norma Brasileira Regulamentadora - NBR 6232, Associação Brasileira de Normas Técnicas - ABNT (2013). Os discos foram retirados a $0,50 \mathrm{~m}$ da base dos moirões, que coincide com a região de afloramento de peças instaladas no solo, e mais propícia ao ataque de organismos xilófagos, como fungos e térmitas (Figura 1). Os mesmos foram pincelados com solução de cromo-azurol S, que revela uma coloração azul escuro intensa na presença de cobre. Para tanto, foi seguida a metodologia descrita por Paes et al. (2014) e Lopes (2012).

Para a determinação da retenção de CCA, foram retirados discos suplementares, na região citada e efetuou-se a digestão úmida das amostras de $1,5 \times 1,0 \times 2,0 \mathrm{~cm}$ (radial x tangencial x longitudinal), em sentidos opostos (Figura 1), seguindo a metodologia descrita por e Lopes (2014) e Paes et al. (2012). 


\section{Ensaios de resistência biológica a térmitas xilófagos}

\section{Ensaio de alimentação forçada}

Este ensaio foi realizado com o térmita subterrâneo Nasutitermes corniger Motsch., conforme o método descrito pela American Society for Testing and Materials - ASTM D - 3345 (ASTM, 2005a). Foram utilizados 96 corpos de prova de 1,5 x 1,0 x 2,0 cm (radial x tangencial x longitudinal), sendo 72 tratados com CCA-C (36 de cada espécie, 18 de cada posição) e 24 não tratados (12 de cada espécie, seis de cada posição), obtidos conforme Figura 1. Os corpos de prova foram secos em estufa a $103 \pm 2{ }^{\circ} \mathrm{C}$ até atingirem massa constante, e sua massa obtida em balança analítica $(0,0001 \mathrm{~g})$.

Para a realização do ensaio foi coletada uma colônia de cupins nas proximidades do município de Jerônimo Monteiro, ES. No Laboratório de Biodeterioração e Proteção da Madeira, Departamento de Ciências Florestais e da Madeira, Universidade Federal do Espírito Santo, a mesma foi depositada sobre uma bandeja para tubetes $(40 \times 70 \mathrm{~cm})$, apoiada sobre dois tijolos de 10 furos, em uma caixa de cimento amianto de $500 \mathrm{~L}$ de capacidade, contendo uma camada de $10 \mathrm{~cm}$ de areia umedecida recoberta por papelão umedecido para facilitar a captura dos cupins. A caixa foi disposta sobre quatro tijolos, depositados em bandeja $(5 \times 20 \times 30 \mathrm{~cm})$ com água para evitar a fuga dos insetos.

O ensaio foi montado em frascos de vidro de $600 \mathrm{~mL}$, com tampa metálica rosqueável, ao seguirem o disposto na ASTM D - 3345 (ASTM, 2005a). Os frascos foram preenchidos com $200 \mathrm{~g}$ de areia lavada, esterilizada em estufa a $103 \pm 2^{\circ} \mathrm{C}$ por 24 horas, com adição de $36 \mathrm{~mL}$ de água destilada, um corpo de prova soterrado até a metade de seu comprimento encostado em uma das paredes do frasco e $1 \pm 0,05 \mathrm{~g}$ da térmita subterrânea (média de 350 térmitas) contendo de $80 \%$ operários e $20 \%$ soldados, proporção existente na colônia.

O ensaio permaneceu em sala climatizada $\left(27 \pm 2^{\circ} \mathrm{C}\right.$ e $65 \pm 5 \%$ de umidade relativa $)$ durante 28 dias. Decorrido o período do ensaio, os corpos de prova foram removidos dos frascos, lavados com água corrente, o resíduo de areia e excrementos dos cupins removidos com escova macia, e secos em estufa, sob as condições já descritas, até atingirem massas constantes, para a determinação da perda de massa dos mesmos.

A resistência à deterioração causada pelos cupins subterrâneos foi avaliada em termos de perda de massa dos corpos de prova, mortalidade dos insetos, e pela análise visual do ataque (desgaste), Tabela 2.

Tabela 2. Avaliação do desgaste da madeira e da mortalidade das térmitas.

Table 2. Wood waste evaluation and mortality of termites.

\begin{tabular}{lc}
\hline Tipo de Desgaste & Nota \\
\hline Sadio, permitindo escarificações superficiais & 10 \\
Ataque superficial & 9 \\
Ataque moderado, havendo penetração & 7 \\
Ataque intenso & 4 \\
Falha, havendo ruptura dos corpos de prova & 0 \\
\hline Mortalidade & $(\%)$ \\
\hline Baixa & $0-33$ \\
Moderada & $34-66$ \\
Alta & $67-99$ \\
Total & 100 \\
\hline
\end{tabular}

Fonte: ASTM D 3345 (ASTM, 2005a).

\section{Ensaio de preferência alimentar}

Este ensaio não é normalizado, mas segundo Supriana (1985) é mais representativo das condições de campo e, assim, sendo utilizado por vários pesquisadores e instituições de pesquisa, para avaliação da resistência biológica da madeira e derivados a térmitas subterrâneas (BRITO, 2015; CALEGARI et al., 2014; MELO, 2009; PAES et al., 2015; 2014, 2011; 2010; 2007a).

Para a realização do ensaio foi coletada uma colônia do térmita Nasutitermes corniger Motsch., nas proximidades do município de Jerônimo Monteiro, ES. Após a coleta, a mesma foi depositada numa caixa de $500 \mathrm{~L}$ de capacidade, contendo areia umedecida a uma altura de aproximadamente $10 \mathrm{~cm}$ do fundo. A caixa foi suportada por quatro tijolos, imersos em recipientes com água, para 
evitar a fuga das térmitas. A colônia foi acomodada sobre uma grelha, apoiada em dois tijolos.

Na montagem do ensaio, seguiu-se um delineamento em blocos casualizados, tendo sido utilizados 96 corpos de prova de 1,5 x 1,0 x 2,0 cm (radial x tangencial x longitudinal) (Figura 1), em que 72 destes foram tratados com CCA (36 de cada espécie, 18 da posição 1 e 18 da posição 2 ) e 24 não tratados (12 de cada espécie, 6 da posição 1 e 6 da posição 2). Os corpos de prova foram secos em estufa a $103 \pm 2^{\circ} \mathrm{C}$ até atingirem massa constante, mensurados em balança analítica (0,0001g de precisão). Na Figura 2 é exemplificado o esquema de montagem deste ensaio.
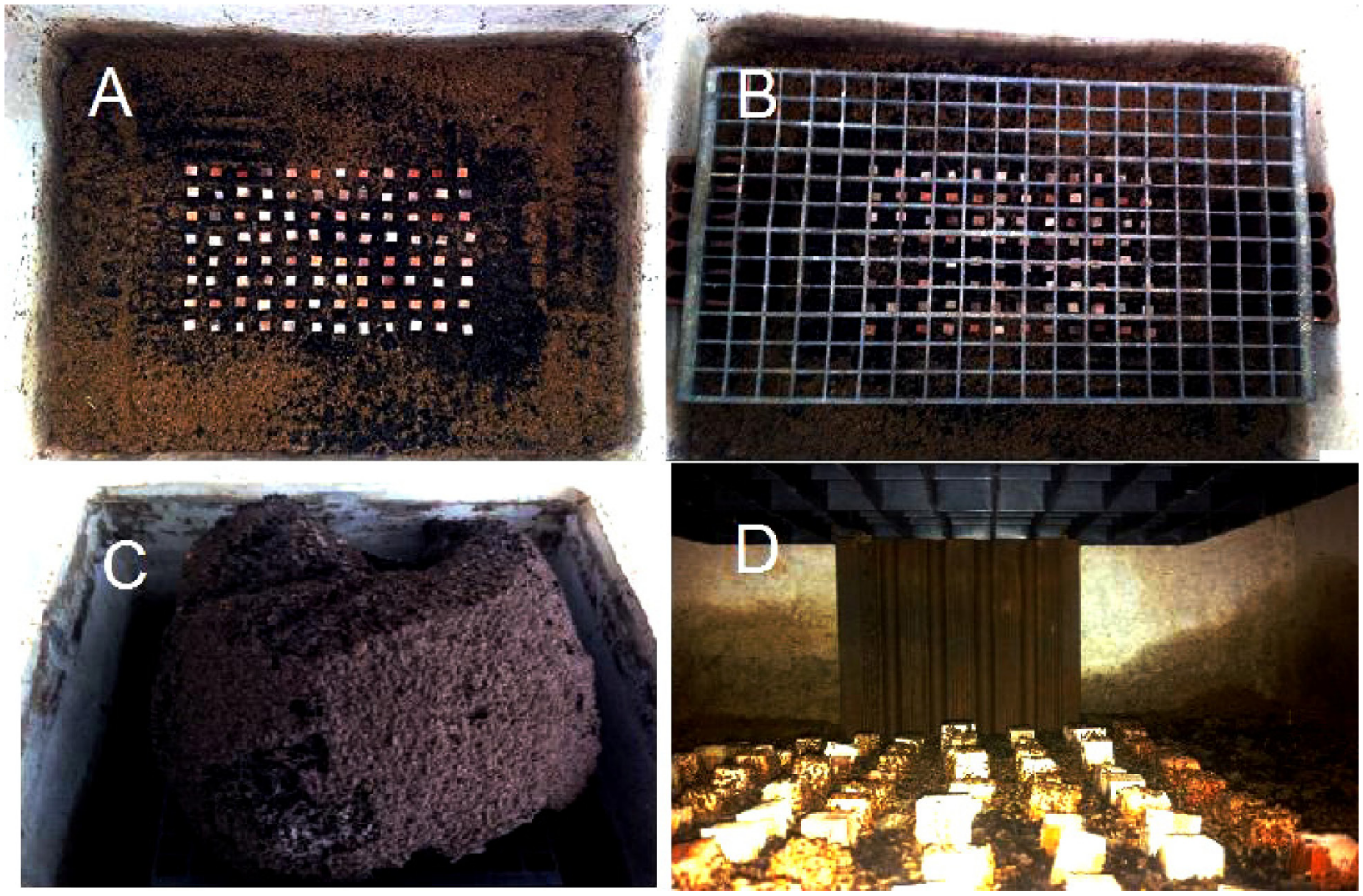

Figura 2. Etapas da montagem do ensaio de preferência alimentar com o térmita Nasutitermes corniger. A $=$ Arranjo dos corpos de prova na caixa. $\mathrm{B}=$ Sobreposição da grelha nos tijolos para suporte da colônia. $\mathrm{C}=$

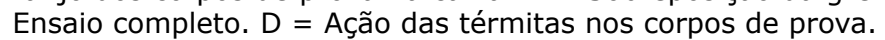

Figure 2. Step of assembly choice feeding test with the termite Nasutitermes corniger. $A=$ Arrangement of the specimens in the box. $B=$ grid overlay on the bricks to support the colony. $C=$ complete test. $D=A$ ttack of termites in the specimens.

Os corpos de prova ficaram expostos à ação das térmitas durante 45 dias e, decorrido o período do ensaio, os mesmos foram removidos da caixa d'água e lavados com água corrente com o uso de escova macia, e tiveram a massa anidra final determinada.

A resistência à deterioração pelos cupins subterrâneos foi avaliada em termos de perda de massa dos corpos de prova, de acordo com a ASTM D - 2017 (2005b) e pela análise visual do ataque (Tabela 2).

\section{Análise estatística dos resultados}

Para a porcentagem de cerne, alburno, relação cerne:alburno e massa específica básica dos moirões foi realizado o teste $t$ de Student para amostras independentes $(\mathrm{p} \leq 0,05)$, considerando $\mathrm{o}$ efeito madeira e da variável resposta em questão.

$\mathrm{Na}$ análise da retenção e ensaio biológico de alimentação forçada foi realizado um delineamento inteiramente casualisado com arranjo fatorial, com os fatores espécie (dois níveis), classe de diâmetro (três níveis) e posição no disco (dois níveis) e para a penetração apenas os fatores espécies e classe de diâmetro foram considerados. No ensaio de preferência alimentar utilizou-se o delineamento em blocos casualizados com arranjo em fatorial, em que as posições no disco foram os blocos, com os fatores espécie (dois níveis) e classe de diâmetro (três níveis).

Para possibilitar a análise estatística, os dados de perda de massa e do desgaste foram transformados em arcoseno $\left(\sqrt{\frac{\text { perda de massa }}{100}}\right)$ e $\sqrt{\text { desgaste }+0,5}$ como sugerido por Steel e Torrie (1980) 
para permitir a normalidade dos dados (teste de Lilliefors) e homogeneidade das variâncias (teste de Cochran). Na análise e avaliação dos ensaios, foi empregado o teste de Tukey $(\mathrm{p} \leq 0,05)$ para as fontes de variação detectadas como significativas pelo teste $\mathrm{F}(\mathrm{p} \leq 0,05)$.

\section{RESULTADOS E DISCUSSÃO}

\section{Relação cerne:alburno e massa específica básica dos moirões}

De acordo com a Tabela 3, a madeira de C. torelliana apresentou maior porcentagem de cerne e, consequentemente, menor de alburno, quando comparada à de E. grandis x E. urophylla. Salienta-se que maiores relações cerne:alburno resultam em menor penetração de produto químico pela madeira, uma vez que o cerne, em virtude de incrustações e tiloses nos vasos, torna-se impermeável a fluídos (OLIVEIRA, 2003), fato constatado neste trabalho.

Tabela 3. Valores médios dos parâmetros avaliados para as madeiras estudadas.

Table 3. Average values of the parameters evaluated for the studied woods.

\begin{tabular}{|c|c|c|}
\hline Parâmetro Avaliado & Corymbia torelliana & E. grandis $x$ E. urophylla \\
\hline$\%$ Cerne & 67,87 a $(19,74)$ & $56,53 \mathrm{~b}(22,89)$ \\
\hline$\%$ Alburno & $32,13 \mathrm{~b}(41,33)$ & 43,47 a $(29,74)$ \\
\hline Relação Cerne:Alburno & 2,65 a $(55,47)$ & $1,51 \mathrm{~b}(53,78)$ \\
\hline \multicolumn{3}{|c|}{ Massa Específica Básica das Madeiras $\left(\mathrm{g} \mathrm{cm}^{-3}\right)$} \\
\hline Posição no Moirão & Corymbia torelliana & E. grandis $x$ E. urophylla \\
\hline Base & 0,54 a $(5,55)$ & $0,51 \mathrm{~b}(5,81)$ \\
\hline Meio & 0,54 a $(5,54)$ & $0,51 \mathrm{~b}(3,91)$ \\
\hline Topo & 0,53 a $(7,47)$ & $0,51 \mathrm{a}(5,85)$ \\
\hline
\end{tabular}

Médias seguidas por letra minúscula, na horizontal, no mesmo parâmetro, não diferem entre si pelo teste t para amostras independentes ( $\mathrm{p}>$ 0,05). Valor entre parênteses corresponde ao coeficiente de variação.

Os moirões da madeira de C. torelliana apresentaram massa específica básica superior a daqueles de E. grandis $\mathrm{x}$ E. urophylla, exceto para as amostras provenientes do topo dos mesmos (Tabela 3). No entanto, tal diferença foi de apenas $0,03 \mathrm{~g} \mathrm{~cm}^{-3}$.

\section{Penetração e retenção do CCA-C nas madeiras}

Conforme Tabela 4, as maiores penetrações foram observadas nos menores diâmetros. Para estes casos, a penetração foi maior na madeira de E. grandis $\mathrm{x}$ E. urophylla. A porcentagem de alburno possivelmente contribuiu para a penetração do produto, em que maiores diâmetros, corresponderam também à maior proporção de cerne, o qual, por ser de madeira juvenil, é pouco resistente à deterioração biológica (PAES; VITAL, 2000).

Tabela 4. Valores médios da penetração de cobre para classe diamétrica e da retenção de CCA-C para posição no disco.

Table 4. Average values of copper penetration to diametric class and of retention of CCA-C for position in the disk.

\begin{tabular}{|c|c|c|c|}
\hline \multirow{2}{*}{ Madeira } & \multicolumn{3}{|c|}{ Penetração (mm) / Classe Diamétrica (cm) } \\
\hline & 8 a 10 & 10 a 12 & 12 a 14 \\
\hline C. torelliana & $\begin{array}{c}18,65 \mathrm{Ab} \\
(35,49)\end{array}$ & $\begin{array}{c}16,45 \mathrm{Bb} \\
(28,14)\end{array}$ & $\begin{array}{c}13,42 \mathrm{Ca} \\
(18,37)\end{array}$ \\
\hline E. urograndis & $\begin{array}{c}22,47 \mathrm{Aa} \\
(20,73)\end{array}$ & $\begin{array}{c}19,80 \mathrm{Ba} \\
(25,30)\end{array}$ & $\begin{array}{c}12,43 \mathrm{Ca} \\
(18,18)\end{array}$ \\
\hline \multirow{2}{*}{ Madeira } & \multicolumn{3}{|c|}{ Retenção (kg i.a m³) / Posição no Disco } \\
\hline & 1 & 2 & \\
\hline Corymbia torelliana & $\begin{array}{c}11,44 \mathrm{Aa} \\
(15,73)\end{array}$ & $\begin{array}{c}1,66 \mathrm{Bb} \\
(140,36)\end{array}$ & \\
\hline E. urograndis & $\begin{array}{c}11,74 \mathrm{Aa} \\
(23,08)\end{array}$ & $\begin{array}{c}4,67 \mathrm{Ba} \\
(68,09)\end{array}$ & \\
\hline
\end{tabular}

Médias seguidas pela mesma letra minúscula, na coluna, e maiúscula, na linha, não diferem entre si (Tukey, p > 0,05). Valor entre parênteses corresponde ao coeficiente de variação.

Observa-se que os maiores valores de retenção do CCA-C foram constatados na posição 1 (tomada de 0 a 1,5 cm da periferia do disco) e não houve diferença entre as espécies para esta posição 
(Tabela 4). No entanto, para a posição $2(1,5$ a 3,0 cm) a quantidade de produto retido foi maior na madeira E. grandis $\mathrm{x}$ E. urophylla. Nota-se também que para esta posição, a retenção foi inferior à estipulada pela NBR 9480 (ABNT, 2009), que é de 6,5 kg i.a m ${ }^{-3}$ de madeira tratada, estando as mesmas susceptíveis ao ataque de xilófagos, principalmente no caso de rachadura na peça, o que poderá expor partes não devidamente protegidas do lenho.

\section{Deterioração causada por térmitas subterrâneos}

\section{Ensaio de alimentação forçada}

Destaca-se que os cupins subterrâneos, em alguns casos, especialmente naqueles em que as amostras não foram tratadas com CCA, fizeram túneis na areia, indicando o vigor dos insetos utilizados, tendo sobrevivido durante nove dias sem a presença de alimento. Para os moirões tratados com CCA, os mesmos tiveram organização antagônica quanto a sua posição e movimentação em relação ao corpo de prova no interior do frasco, indicando o efeito repelente do preservativo, como preconiza a ASTM D - 3345 (2005a).

A posição 2 (1,5 a 3,0 cm para o interior do disco) obteve a maior perda de massa (Tabela 5), uma vez que foram corpos de prova constituídos, na sua maior parte, de cerne, em que o produto preservativo não penetrou na madeira, não conferindo proteção à mesma. Destaca-se, que em função da idade das árvores (Tabela 1), o lenho era juvenil, sem a resistência proporcionada pela madeira adulta.

Tabela 5. Valores médios da perda de massa para posição no disco.

Table 5. Average mass loss values for position in the disc.

\begin{tabular}{cc}
\hline Posição no Disco & Perda de Massa (\%) \\
\hline \multirow{2}{*}{2} & $5,68 \mathrm{~b}$ \\
& $(103,52)$ \\
& $9,58 \mathrm{a}$ \\
& $(87,57)$ \\
\hline
\end{tabular}

Médias seguidas pela mesma letra minúscula, na coluna, não diferem entre si, pelo teste de Tukey $(p>0,05)$. Valor entre parênteses corresponde ao coeficiente de variação.

Quando foram comparadas as perdas de massa causadas pelos cupins, para a posição 1 nas madeiras tratadas, as mesmas foram semelhantes as do cerne de $C$. maculata que obteve perda de massa média de 5,05\% (PAES; VITAL, 2000). Para a posição $2(1,5$ a 3,0 cm) as perdas de massa, neste trabalho, foram superiores as das madeiras de alburno de C. citriodora, C. maculata e E. cloeziana $(8,21 ; 6,81 ; 7,75 \%$, respectivamente), no entanto, bem inferiores a das madeiras de cerne interno de E. saligna $(20,73 \%)$ e E. urophylla $(25,76 \%)$ (PAES; VITAL, 2000). Isto comprova que os valores de retenção obtidos (Tabela 4), foram capazes de proporcionar melhorias na resistência da porção interna (posição 2) das madeiras tratadas.

Nota-se (Tabela 6) aumento de resistência ao ataque de térmitas xilófagas quando as madeira foram tratadas com CCA e que, para aquelas não tratadas, não houve diferença entre as perdas de massa, contudo, a de C. torelliana, foi a mais atacada pelos cupins.

Tabela 6. Valores médios da perda de massa das madeiras para classes diamétricas.

Table 6. Average values of mass loss of woods for diametric classes.

\begin{tabular}{lccc}
\hline \multirow{2}{*}{ Madeira } & \multicolumn{3}{c}{ Perda de Massa (\%) / Classe Diamétrica (cm) } \\
\cline { 2 - 4 } & $\mathbf{8}$ a 10 & $\mathbf{1 0}$ a 12 & $\mathbf{1 2}$ a 14 \\
\hline Não tratada & $25,58 \mathrm{aA}$ & $16,30 \mathrm{aB}$ & $14,56 \mathrm{aB}$ \\
C. torelliana & $(46,20)$ & $(21,10)$ & $(6,79)$ \\
\hline Não tratada & $19,69 \mathrm{aA}$ & $15,78 \mathrm{aA}$ & $13,40 \mathrm{aA}$ \\
E. grandis x E. urophylla & $(25,79)$ & $(15,96)$ & $(20,07)$ \\
\hline Tratada & $4,97 \mathrm{bA}$ & $7,99 \mathrm{bA}, 65 \mathrm{bA}$ \\
C. torelliana & $(47,20)$ & $(79,09)$ & $(83,71)$ \\
\hline Tratada & $2,36 \mathrm{bA}$ & $2,51 \mathrm{cA}$ & $2,46 \mathrm{cA}$ \\
E. grandis x E. urophylla & $(71,18)$ & $(74,10)$ & $(86,99)$
\end{tabular}

Médias seguidas pela mesma letra minúscula, na coluna, e maiúscula, na linha, não diferem entre si, pelo teste de Tukey $(p>0,05)$. Valor entre parênteses corresponde ao coeficiente de variação. 
De maneira geral, os moirões das madeiras tratada e não tratada de E. grandis x E. urophylla foram mais resistentes ao cupim xilófago N. corniger, quando comparados aos provenientes de peças tratadas e não tratadas de C. torelliana, para todas as classes diamétricas estudadas, embora não tenha diferido, estatisticamente, na classe de 8 a $10 \mathrm{~cm}$ dos moirões tratados. Isto pode ser explicado pela diferença de retenção na posição $2(1,5$ a 3,0 cm) nos moirões de E. grandis x E. urophylla, quando comparados áqueles da madeira de C. torelliana.

A vitalidade das térmitas nos frascos foi avaliada diariamente, durante os 28 dias, como preconizado para este ensaio (ASTM D - 3345, 2005a). Para as amostras tomadas nas posições 1 e 2 dos moirões tratados e não tratados foi avaliada a mortalidade dos cupins ao fim do ensaio e, apenas para os tratados, os dias necessários para a morte dos insetos (Tabela 7).

Tabela 7. Valores médios da mortalidade, tempo necessário para a morte das térmitas nas madeiras tratadas e não tratadas.

Table 7. Average mortality values, time required for death of the treated wood termites and untreated wood.

\begin{tabular}{|c|c|c|c|c|}
\hline \multirow{3}{*}{ Mortalidade (\%) } & \multicolumn{2}{|c|}{ Madeiras não Tratadas } & \multicolumn{2}{|c|}{ Madeiras Tratadas } \\
\hline & $\begin{array}{l}\text { Corymbia } \\
\text { torelliana }\end{array}$ & $\begin{array}{l}\text { E. grandis } \mathrm{x} \\
\text { E. urophylla }\end{array}$ & $\begin{array}{l}\text { Corymbia } \\
\text { torelliana }\end{array}$ & $\begin{array}{l}\text { E. grandis } \mathbf{x} \\
\text { E. urophylla }\end{array}$ \\
\hline & 50 & 55 & 95 & 100 \\
\hline & \multicolumn{4}{|c|}{ Madeiras Tratadas } \\
\hline \multirow[t]{2}{*}{ Tempo (dias) } & \multicolumn{2}{|c|}{ Corymbia torelliana } & \multicolumn{2}{|c|}{ E. grandis $x$ E. urophylla } \\
\hline & \multicolumn{2}{|c|}{13} & \multicolumn{2}{|c|}{9} \\
\hline
\end{tabular}

Observa-se que foram obtidas mortalidades consideradas moderadas pela ASTM D - 3345 (2005a) para ambas as madeiras não tratadas (Tabela 7). Em nenhum caso foi relatado mortalidade total dos cupins em contato com as amostras obtidas de tais moirões. Desta forma, pode-se afirmar que os mesmos apresentam susceptibilidade moderada à deterioração pelo $N$. corniger.

O tempo médio necessário para a morte dos cupins foi de 13 e 9 dias, para as madeiras de $C$. torelliana e de E. grandis x E. urophylla, respectivamente. Destaca-se que em quatro amostras de C. torelliana (posição 2, tomada de 1,5 a 3,0 cm da periferia da peça), os insetos foram capazes de resistir à toxidez do CCA-C, em decorrência da menor retenção do produto e da presença de cerne nas amostras. Isto reforça o que foi discutido anteriormente, que a madeira de cerne juvenil não confere resistência aos cupins.

Para a madeira de C. torelliana ocorreu 95\% de morte dos cupins ao fim dos 28 dias de ensaio, com os demais resistindo ao efeito do CCA-C. Destaca-se a efetividade do CCA-C, ocasionando a morte em $100 \%$ das amostras (posição 1 e 2) para a madeira de E. grandis $\mathrm{x}$ E. urophylla ao fim dos 28 dias de ensaio.

Para a análise conjunta das duas espécies e situações (madeira tratada e não tratada), observa-se (Tabela 8), que a menor nota foi atribuída às amostras da posição $2(1,5 \mathrm{a} \mathrm{3,0} \mathrm{cm})$, nos moirões de classe diamétrica de 10 a $12 \mathrm{~cm}$, diferindo estatisticamente das demais classes de diâmetro. Desgaste semelhante foi encontrado para a resistência natural das madeiras de louro pardo (Cordia trichotoma), jurema preta (Mimosa tenuiflora), sabiá (Mimosa caesalpiniifolia) e teca (Tectona grandis), classificadas como resistentes ao ataque de N. corniger (Paes et al., 2007b).

Tabela 8. Notas médias do desgaste por classe diamétrica e posição no disco.

Table 8. Average notes of waste by diametric class and position in the disk.

\begin{tabular}{lcc}
\hline \multirow{2}{*}{ Classe Diamétrica $(\mathbf{c m})$} & \multicolumn{2}{c}{ Posição no Disco / Desgaste (Nota) } \\
\cline { 2 - 3 } & $\mathbf{1}$ & $\mathbf{2}$ \\
\hline \multirow{2}{*}{8 a 10} & $8,25 \mathrm{aA}$ & $8,11 \mathrm{aA}$ \\
& $(29,93)$ & $(33,16)$ \\
\hline \multirow{2}{*}{10 a 12} & $8,38 \mathrm{aA}$ & $6,97 \mathrm{bB}$ \\
& $(26,61)$ & $(38,30)$ \\
\hline \multirow{2}{*}{12 a 14} & $8,73 \mathrm{aA}$ & $7,91 \mathrm{aB}$ \\
\end{tabular}

Médias seguidas pela mesma letra minúscula, na coluna, e maiúscula, na linha, não diferem entre si, pelo teste de Tukey ( $\mathrm{p}>0,05)$. Valor entre parênteses corresponde ao coeficiente de variação. 
De acordo com a Tabela 9, independente do diâmetro analisado, as maiores notas foram atribuídas às amostras provenientes da posição 1 (profundidades de 0 a 1,5 cm), diferindo estatisticamente entre as mesmas, com exceção do diâmetro de 8 a $10 \mathrm{~cm}$.

De acordo com a Tabela 9, observou-se que as melhores notas foram atribuídas às madeiras tratadas e, em valores médios absolutos, o E. grandis $\mathrm{x}$ E. urophylla atingiu as maiores notas. Em termos de durabilidade natural, ambas as espécies obtiveram resistências semelhantes ao térmita N. corniger.

Tabela 9. Notas médias do desgaste por classe diamétrica para cada madeira ensaiada.

Table 9. Average notes of waste by diametric class for each tested wood.

\begin{tabular}{lccc}
\hline \multirow{2}{*}{ Madeiras } & \multicolumn{3}{c}{ Classe Diamétrica (cm) / Desgaste (Nota) } \\
\cline { 2 - 4 } & $\mathbf{8} \mathbf{~ a ~ 1 0}$ & $\mathbf{1 0} \mathbf{a} \mathbf{1 2}$ & $\mathbf{1 2}$ a 14 \\
\hline Tratada & $9,48 \mathrm{aA}$ & $8,45 \mathrm{aB}$ & $8,83 \mathrm{bA}$ \\
C. torelliana & $(8,54)$ & $(25,08)$ & $(19,02)$ \\
\hline Tratada & $9,61 \mathrm{aA}$ & $9,15 \mathrm{aA}$ & $9,71 \mathrm{aA}$ \\
E. grandis x E. urophylla & $(6,86)$ & $(11,69)$ & $(4,63)$ \\
\hline Não tratada & $3,75 \mathrm{bB}$ & $3,90 \mathrm{bB}$ & $5,35 \mathrm{cA}$ \\
C. torelliana & $(3,84)$ & $(41,28)$ & $(28,59)$ \\
\hline Não tratada & $4,4 \mathrm{bA}$ & $4,75 \mathrm{bA}$ & $5,6 \mathrm{cA}$ \\
E. grandis x E. urophylla & $(36,36)$ & $(28,00)$ & $(30,17)$ \\
\hline
\end{tabular}

Médias seguidas pela mesma letra minúscula, na coluna, e maiúscula, na linha, não diferem entre si, pelo teste de Tukey $(p>0,05)$. Valor entre parênteses corresponde ao coeficiente de variação.

Em ambas as situações, ou seja, madeiras tratada e não tratada, as piores notas foram obtidas na posição 2 (1,5 a 3,0 cm), entretanto, não diferindo estatisticamente entre profundidades para as madeiras não tratadas (Tabela 10). Para as madeiras tratadas ocorreu diferença entre as profundidades apenas para a madeira de C. torelliana, em virtude, principalmente, da maior relação cerne:alburno.

Tabela 10. Notas médias do desgaste para posição no disco para cada madeira ensaiada.

Table 10. Average notes of waste for position in the disk for each tested wood.

\begin{tabular}{lcc}
\hline \multirow{2}{*}{ Madeiras } & \multicolumn{2}{c}{ Posição no Disco (cm) / Desgaste (Nota) } \\
\cline { 2 - 3 } & $\mathbf{1}$ & $\mathbf{2}$ \\
\hline Tratada & $9,57 \mathrm{aA}$ & $8,26 \mathrm{bB}$ \\
C. torelliana & $(7,41)$ & $(25,06)$ \\
\hline Tratada & $9,65 \mathrm{aA}$ & $9,33 \mathrm{aA}$ \\
E. grandis x E. urophylla & $(6,21)$ & $(10,07)$ \\
\hline Não tratada & $4,7 \mathrm{bA}$ & $3,96 \mathrm{cA}$ \\
C. torelliana & $(27,44)$ & $(48,73)$ \\
\hline Não tratada & $5,26 \mathrm{bA}$ & $4,56 \mathrm{cA}$ \\
E. grandis x E. urophylla & $(30,60)$ & $(33,77)$ \\
\hline
\end{tabular}

Médias seguidas pela mesma letra minúscula, na coluna, e maiúscula, na linha, não diferem entre si, pelo teste de Tukey $(p>0,05)$. Valor entre parênteses corresponde ao coeficiente de variação.

\section{Ensaio de preferência alimentar}

Como observado (Tabela 11), a posição 1 ( 0 a 1,5 cm de retirada dos corpos de prova das madeiras tratadas) foi a que resultou em menor perda de massa. Este resultado foi obtido em virtude desta posição possuir a maior retenção de produto preservativo, conferindo melhor resistência à madeira. A perda de massa mais elevada, na posição $2(1,5$ a 3,0 cm), pode ser explicada pela pouca penetração e retenção do CCA-C em regiões próximas ou de transição de alburno cerne.

Para as madeiras tratadas foram registrados os menores valores médios de perda de massa para o E. grandis $\mathrm{x}$ E. urophylla. Isto pode ser explicado, principalmente, pela menor relação cerne:alburno, que promoveram as maiores retenções de CCA. Evidencia-se o ganho de resistência das madeiras tratadas, em comparação às não tratadas, quando submetidas ao ataque de $N$. corniger, uma vez que a perda de massa causada foi, aproximadamente, de $94,76 \%$ e $23,44 \%$ menor para as posições 1 $(0$ a $1,5 \mathrm{~cm})$ e $2(1,5$ a 3,0) respectivamente, para o C. torelliana e, $95,15 \%$ e $47,87 \%$ menor para as posições 1 e 2, respectivamente, para o E. grandis x E. urophylla. 
Tabela 11. Valores médios da perda de massa para posição no disco e madeira ensaiada.

Table 11. Average values of the mass loss for position in the disk and tested wood.

\begin{tabular}{lcc}
\hline \multirow{2}{*}{ Madeira } & \multicolumn{1}{c}{ Perda de Massa (\%) / Posição no Disco } \\
\cline { 2 - 3 } & $\mathbf{1}$ & $\mathbf{2}$ \\
\hline Tratada & $5,24 \mathrm{bB}$ & $76,56 \mathrm{aA}$ \\
C. torelliana & $(70,22)$ & $(42,99)$ \\
\hline Tratada & $4,85 \mathrm{bB}$ & $49,92 \mathrm{bA}$ \\
E. grandis x E. urophylla & $(158,55)$ & $(77,14)$ \\
\hline Não tratada & $100 \mathrm{aA}$ & $100 \mathrm{aA}$ \\
C. torelliana & $(0)$ & $(0)$ \\
\hline Não tratada & $100 \mathrm{aA}$ & $97,79 \mathrm{aA}$ \\
E. grandis x E. urophylla & $(0)$ & $(5,63)$
\end{tabular}

Médias seguidas pela mesma letra minúscula, na coluna, e maiúscula, na linha, não diferem entre si, pelo teste de Tukey $(p>0,05)$. Valor entre parênteses corresponde ao coeficiente de variação.

Observa-se que os parâmetros utilizados no tratamento com CCA-C nas madeiras foram eficazes contra $N$. corniger, uma vez que, causaram as menores perdas de massa nas madeiras tratadas. Nota-se que os moirões não tratados de C. torelliana possuíram máxima perda de massa causada pelo cupim xilófago, não diferindo, estatisticamente, daqueles da madeira não tratada de E. grandis x E. urophylla. Paes et al. (2013) obtiveram para a madeira de E. camaldulensis, de quatro anos, não tratada, perdas de massa próximas às encontradas neste trabalho.

O ganho de resistência da madeira proporcionado pelo tratamento com CCA-C foi capaz de conferir, à mesma, comportamento semelhante ao dos lenhos de marmeleiro preto (Croton sonderianus) e nim indiano (Azadirachta indica), classificadas como de resistência moderada (PAES et al., 2007b). Segundo os autores citados, a resistência das espécies florestais, está relacionada à classe (qualidade) e quantidade dos extrativos presentes em cada uma delas.

De maneira geral, a madeira de E. grandis $\mathrm{x}$ E. urophylla obteve as melhores notas de desgaste dos corpos de prova (Tabela 12), com exceção dos moirões de 8 a $10 \mathrm{~cm}$. Para os moirões tratados de $C$. torelliana as notas atribuídas aos corpos de prova diminuíram com o aumento da classe diamétrica, em que a de 8 a $10 \mathrm{~cm}$ diferiu das demais. Para a madeira tratada de E. grandis $\mathrm{x}$ E. urophylla houve semelhança entre os resultados de desgaste entre classes diamétricas avaliadas.

Tabela 12. Valores médios do desgaste por classe diamétrica, posição no disco e madeira ensaiada.

Table 12. Average values of waste by diametric class, position in the disk and tested wood.

\begin{tabular}{lccc}
\hline Madeira & \multicolumn{3}{c}{ Desgaste (Nota) / Classe Diamétrica (cm) } \\
\cline { 2 - 4 } & $\mathbf{8}$ a 10 & $\mathbf{1 0}$ a 12 & $\mathbf{1 2}$ a 14 \\
\hline Tratada & $\begin{array}{c}5,85 \mathrm{aA} \\
(61,70)\end{array}$ & $\begin{array}{c}3,65 \mathrm{bB} \\
(106,30)\end{array}$ & $\begin{array}{c}3,63 \mathrm{bB} \\
(104,40)\end{array}$ \\
\hline $\begin{array}{l}\text { C. torelliana } \\
\text { E. gratada }\end{array}$ & $\begin{array}{l}5,78 \mathrm{aA} \\
(65,05)\end{array}$ & $\begin{array}{c}5,65 \mathrm{aA} \\
(65,13)\end{array}$ & $\begin{array}{c}6,53 \mathrm{aA} \\
(56,20)\end{array}$ \\
\hline $\begin{array}{l}\text { Não tratada } \\
\text { C. torelliana }\end{array}$ & $0 \mathrm{bA}$ & $0 \mathrm{cA}$ & $0 \mathrm{cA}$ \\
\hline $\begin{array}{l}\text { Não tratada } \\
\text { E. grandis x E. urophylla }\end{array}$ & $0 \mathrm{bA}$ & $0 \mathrm{cA}$ & $0 \mathrm{cA}$ \\
\hline
\end{tabular}

\begin{tabular}{|c|c|c|}
\hline \multirow{2}{*}{ Madeira } & \multicolumn{2}{|c|}{ Desgaste (Nota) / Posição no disco } \\
\hline & 1 & 2 \\
\hline $\begin{array}{l}\text { Tratada } \\
\text { C. torelliana } \\
\end{array}$ & $\begin{array}{c}7,7 a A \\
(22.33) \\
\end{array}$ & $\begin{array}{l}1,05 \mathrm{bB} \\
(214,28)\end{array}$ \\
\hline $\begin{array}{l}\text { Tratada } \\
\text { E. grandis } x \text { E. urophylla }\end{array}$ & $\begin{array}{l}8,8 \mathrm{aA} \\
(9,43)\end{array}$ & $\begin{array}{l}3,17 \mathrm{aA} \\
(104,10)\end{array}$ \\
\hline $\begin{array}{l}\text { Não tratada } \\
\text { C. torelliana }\end{array}$ & $0 \mathrm{bA}$ & $0 \mathrm{cA}$ \\
\hline $\begin{array}{l}\text { Não tratada } \\
\text { E. grandis } x \text { E. urophylla }\end{array}$ & $0 \mathrm{bA}$ & $0 \mathrm{cA}$ \\
\hline
\end{tabular}

Médias seguidas pela mesma letra minúscula, na coluna, e maiúscula, na linha, para cada parâmetro avaliado, não diferem entre si, pelo teste de Tukey $(p>0,05)$. Valor entre parênteses corresponde ao coeficiente de variação.

Nota-se que as piores notas foram observadas nos moirões não tratados de ambas as espécies, em todas as classes diamétricas estudadas, diferindo das madeiras tratadas, comprovando a efi- 
ciência dos parâmetros utilizados no tratamento industrial (concentração da solução, tempo e pressão de tratamento).

Ao analisar o desdobramento da interação espécie e híbrido x posição no disco, observa-se que o melhor resultado das notas está associado à presença de produto preservativo, ou seja, posição 1 (0 a 1,5cm), conferindo maior resistência à madeira, haja vista que as madeiras não tratadas tiveram as piores notas de desgaste.

O ganho de resistência das madeiras tratadas, ao analisar o desgaste dos corpos de prova, de acordo com a posição 1, pode ser comparada aos do lenhos de louro pardo, jurema preta e sabiá, classificados como resistentes (PAES et al., 2007b). Ressalta-se que, em estudos de perda de massa e desgaste (nota) atribuídos aos corpos de prova, as maiores notas estão relacionadas com as menores perdas de massa causada por N. corniger.

\section{CONCLUSÕES}

Os parâmetros utilizados para o tratamento das madeiras de C. torelliana e E. grandis x E. urophylla foram eficazes para proporcionar elevada resistência à térmita xilófaga nos ensaios de alimentação forçada e preferência alimentar.

A relação cerne:alburno exerce influência nas propriedades de durabilidade da madeira no sentido inversamente proporcional, ou seja, quanto menor esta relação melhores características de durabilidade serão alcançadas, proporcionada pela melhor penetração e retenção do CCA-C na madeira.

A madeira tratada de E. grandis $\mathrm{x}$ E. urophylla obteve as menores perdas de massa em ambos os ensaios com N. corniger e, também, as maiores notas (desgaste) nos ensaios de alimentação forçada e preferência alimentar.

O tratamento preservativo foi eficiente em garantir que a parte tratável (alburno) dos moirões de ambas as espécies florestais testadas, não fosse atacado pelos cupins, levando os mesmos a consumirem a madeira de cerne não impregnada pelo CCA-C.

\section{REFERÊNCIAS BIBLIOGRÁFICAS}

ABNT - ASSOCIAÇÃO BRASILEIRA DE NORMAS TÉCNICAS. NBR 6232: penetração e retenção de preservativos em postes de madeira. Rio de Janeiro, 2013. 16 p.

ABNT - ASSOCIAÇÃO BRASILEIRA DE NORMAS TÉCNICAS. NBR 9480: peças roliças preservadas de eucalipto para construções rurais - Requisitos. Rio de Janeiro, 2009. 12 p.

ASTM - AMERICAN SOCIETY FOR TESTING AND MATERIALS. ASTM D - 3345: standard method for laboratory evaluation of the wood and other cellulosic materials for resistance to termite. West Conshohocken, 2005a. 3 p.

ASTM - AMERICAN SOCIETY FOR TESTING AND MATERIALS. ASTM D - 2017: accelerated laboratory test of natural decay resistance of woods. West Conshohocken, 2005b. 5 p.

BAKER, P. B.; MARCHOSKY JUNIOR, R. J. Arizona termites of economic importance. Tucson: University of Arizona, College of Agriculture and Life Science, 2005, 20 p.

BRITO, J. P. Ação de cupins de madeira seca (Cryptotermes brevis) e de solo (Nasutitermes corniger) nas madeiras termorretificadas de Pinus taeda e de Corymbia citriodora. 2015. 103 p. Tese (Doutorado em Recursos Florestais) - Universidade de São Paulo, Escola Superior de Agricultura "Luiz de Queiroz", Piracicaba, 2015.

CALEGARI, L.; LOPES, P. J. G.; SANTANA, G. M.; STANGERLIN, D. M.; OLIVEIRA, E.; GATTO, D. A. Eficiência de extrato tânico combinado ou não com ácido bórico na proteção da madeira de Ceiba pentandra contra cupim xilófago. Floresta, Curitiba, v. 44, n. 1, p. 43 - 52, 2014. 
Lopes et al. - Resistência biológica da madeira de eucalipto tratada com CCA-C a cupins subterrâneos em laboratório

CLAUSEN, C. A. Biodeterioration of wood. In: Wood handbook: wood as an engineering material. 100. ed. Madison: USDA Forest Service, 2010. p.16 (General Technical Report FPL-GTR-190).

ELEOTÉRIO, E. S. R. Levantamento e identificação de cupins (Insecta: Isoptera) em área urbana de Piracicaba, SP. 2000. 101 p. Dissertação (Mestrado em Ciências) - Universidade de São Paulo, Escola Superior de Agricultura "Luiz de Queiroz", Piracicaba, 2000.

IBA - INDÚSTRIA BRASILEIRA DE ÁRVORES. Anuário estatístico da IBÁ 2014: ano base 2013. Brasília: IBÁ, 2014. 100 p.

IBA - INDÚSTRIA BRASILEIRA DE ÁRVORES. Anuário estatístico da IBÁ 2015: ano base 2014. Brasília: IBÁ, 2015. 80 p.

LOPES, D. J. V. Eficácia dos parâmetros do tratamento industrial na tratabilidade e durabilidade de madeiras de eucalipto. 2014. 79 p. Dissertação (Mestrado em Ciências Florestais) - Universidade Federal do Espírito Santo, Jerônimo Monteiro, 2014.

MELO, R. R. Propriedades físico-mecânicas e resistência a biodeterioradores de chapas aglomeradas constituídas por diferentes proporções de madeira e casca de arroz. 2009. 77 p. Dissertação (Mestrado em Engenharia Florestal) - Universidade Federal de Santa Maria, Santa Maria, 2009.

OLIVEIRA, J. S. Efeito de pré-tratamentos de água quente e congelamento na secagem e propriedades da madeira de Eucalyptus grandis Hill ex Maiden. 2003. 98 p. Dissertação (Mestrado em Engenharia Florestal) - Universidade Federal de Santa Maria, Santa Maria, 2003.

PAES, J. B.; VITAL, B. R. Resistência natural da madeira de cinco espécies de eucalipto a cupins subterrâneos em testes de laboratório Revista Árvore, Viçosa, v. 24, n. 77, p. 97-104, 2000.

PAES, J. B.; SEGUNDINHO, P. G. A.; EUfLOSINO, A. E. R.; SILVA, M. R.; CALIL JUNIOR, C.; OLIVEIRA, J. G. L. Resistance of thermally treated woods to Nasutitermes corniger in a food preference test. Madera y Bosques, Xalapa, v. 21, n. 1, p.139-156, 2015.

PAES, J. B.; MELO, R. R.; GUEDES, R. S.; SOUZA, P. F. Eficiência da madeira de leucena (Leucaena leucocephala) tratatada com CCB contra cupins xilófagos em ensaio de laboratório. Floresta e Ambiente, Seropédica, v. 21, n. 3, p. 376-38, 2014.

PAES, J. B.; MEDEIROS NETO, P. N.; LIMA, C. R.; FREITAS, M. F.; DINIZ, C. E. F. Efeitos dos extrativos e cinzas na resistência natural de quatro madeiras a cupins xilófagos. Cerne, Lavras, v. 19, n. 3, p. 399-405, 2013.

PAES, J. B.; LOMBARDI, L. R.; OLIVEIRA, J. T. S.; SILVA, L. F.; SANTOS, L. L. Qualidade de moirões de eucalipto tratados comercializados em três municípios do Espírito Santo. Floresta e Ambiente, Seropédica, v. 19, n.4, p. $475-482,2012$.

PAES, J. B.; SOUZA, A. D.; LIMA, C. R.; MEDEIROS NETO, P. N. Eficiência dos óleos de nim (Azadirachta indica) e de mamona (Ricinus communis) na proteção da madeira de sumaúma (Ceiba pentandra) contra cupins xilófagos em ensaio de preferência alimentar. Revista Árvore, Viçosa, v. 35, n. 3, p. 751 - 758, 2011.

PAES, J. B.; FONSÊCA, C. M. B.; LIMA, C. R.; SOUZA, A. D. Eficiência do óleo de candeia na melhoria da resistência da madeira de sumaúma a cupins. Cerne, Lavras, v. 16, n. 2, p. 217-225, 2010.

PAES, J. B.; MELO, R. R.; LIMA, C. R; OLIVEIRA, E. Resistência natural de sete madeiras ao cupim subterrâneo Nasutitermes corniger em ensaio de preferência alimentar. Revista Brasileira de Ciências Agrárias, Recife, v. 2, n. 1, p. 57-62, 2007a. 
PAES, J. B.; MELO, R. F.; LIMA, C. R. Resistência natural de sete madeiras a fungos e cupins xilófagos em condições de laboratório, Cerne, Lavras, v. 13, n. 2, p. 160-169, $2007 \mathrm{~b}$.

RICHARDSON, B. A. Wood preservation. 2. ed. New York: E \& FN Spon, 1993. 239 p.

SILVA, V. S. G. Comportamento de forrageamento de Nasutitermes corniger (Motschulsky) (Isoptera: Termitidae) e sua ocorrência em áreas urbanas. 2008. 108 p. Tese (Doutorado em Entomologia) Universidade Estadual do Norte Fluminense Darcy Ribeiro, Campos dos Goytacazes, 2008.

STEEL, R. G. D.; TORRIE, J. H. Principles and procedures of statistic: a biometrical approach. 2. ed. New York: McGraw-Hill, 1980. 633 p.

SUPRIANA, N. Notes the resistance of tropical wood against termites. Stockholm: The International Research Group on Wood Preservation, 1985. 9 p. (Doc. IRG/WP, 1249).

VITAL, B. R. Métodos de determinação da densidade da madeira. Viçosa: SIF, 1984. 21 p. (Boletim Técnico, 1).

Recebido em 20/07/2016

Aceito para publicação em 04/04/2017

Sci. For., Piracicaba, v. 45, n. 115, p. 467-479, set. 2017 DOI: dx.doi.org/10.18671/scifor.v45n115.05 
